\title{
Clinical predictors of vestibulo-ocular dysfunction in pediatric sports-related concussion
}

\author{
Michael J. Ellis, MD, FRCSC, ${ }^{1-5}$ Dean M. Cordingley, MSc, ${ }^{4}$ Sara Vis, ${ }^{4}$ \\ Karen M. Reimer, BMR PT, MSc, ${ }^{4}$ Jeff Leiter, PhD, ${ }^{1,4}$ and Kelly Russell, PhD ${ }^{2,5}$
}

Departments of ${ }^{1}$ Surgery and ${ }^{2}$ Pediatrics and Child Health, and ${ }^{3}$ Section of Neurosurgery, University of Manitoba, ${ }^{4} \mathrm{Pan}$ Am Concussion Program, and ${ }^{5}$ Children's Hospital Research Institute of Manitoba, Canada North Concussion Network, Winnipeg, Manitoba, Canada

\begin{abstract}
OBJECTIVE There were 2 objectives of this study. The first objective was to identify clinical variables associated with vestibulo-ocular dysfunction (VOD) detected at initial consultation among pediatric patients with acute sports-related concussion (SRC) and postconcussion syndrome (PCS). The second objective was to reexamine the prevalence of VOD in this clinical cohort and evaluate the effect of VOD on length of recovery and the development of PCS.
\end{abstract}

METHODS A retrospective review was conducted for all patients with acute SRC and PCS who were evaluated at a pediatric multidisciplinary concussion program from September 2013 to May 2015. Acute SRS was defined as presenting < 30 days postinjury, and PCS was defined according to the International Classification of Diseases, 10th Revision criteria and included being symptomatic 30 days or longer postinjury. The initial assessment included clinical history and physical examination performed by 1 neurosurgeon. Patients were assessed for VOD, defined as the presence of more than 1 subjective vestibular and oculomotor complaint (dizziness, diplopia, blurred vision, etc.) and more than 1 objective physical examination finding (abnormal near point of convergence, smooth pursuits, saccades, or vestibulo-ocular reflex testing). Poisson regression analysis was used to identify factors that increased the risk of VOD at initial presentation and the development of PCS.

RESULTS Three hundred ninety-nine children, including 306 patients with acute SRC and 93 with PCS, were included. Of these patients, $30.1 \%$ of those with acute SRC (65.0\% male, mean age 13.9 years) and $43.0 \%$ of those with PCS (41.9\% male, mean age 15.4 years) met the criteria for VOD at initial consultation. Independent predictors of VOD at initial consultation included female sex, preinjury history of depression, posttraumatic amnesia, and presence of dizziness, blurred vision, or difficulty focusing at the time of injury. Independent predictors of PCS among patients with acute SRC included the presence of VOD at initial consultation, preinjury history of depression, and posttraumatic amnesia at the time of injury.

CONCLUSIONS This study identified important potential risk factors for the development of VOD following pediatric SRC. These results provide confirmatory evidence that VOD at initial consultation is associated with prolonged recovery and is an independent predictor for the development of PCS. Future studies examining clinical prediction rules in pediatric concussion should include VOD. Additional research is needed to elucidate the natural history of VOD following SRC and establish evidence-based indications for targeted vestibular rehabilitation.

http://thejns.org/doi/abs/10.3171/2016.7.PEDS16310

KEY WORDS sports-related concussion; vestibulo-ocular dysfunction; postconcussion syndrome; predictor; trauma

$\mathrm{S}$ PORTS-RELATED concussion (SRC) results from the transmission of abnormal biomechanical forces to the brain leading to temporary alterations in neurological functioning. Most adult athletes achieve full neurological recovery in $1-2$ weeks, ${ }^{20,24}$ but the natural history of pediatric concussion remains poorly understood.
Despite adequate physical and cognitive rest, a significant proportion of pediatric SRC patients can exhibit persistent symptoms leading to prolonged recovery or a diagnosis of postconcussion syndrome (PCS) ${ }^{2,8,11,16,40}$ Previous studies have identified important clinical variables associated with an elevated risk of prolonged recovery and PCS de-

ABBREVIATIONS ADHD = attention deficit hyperactivity disorder; $\mathrm{Cl}=$ confidence interval; ICD-10 = International Classification of Diseases, 10 th Revision; IQR = interquartile range; $L O C=$ loss of consciousness; NPC = near-point convergence; PCS = postconcussion syndrome; PCSS = Post-Concussion Symptom Scale; RR = risk ratio; $\mathrm{SRC}=$ sports-related concussion; VOD = vestibulo-ocular dysfunction; VOR = vestibulo-ocular reflex.

SUBMITTED June 1, 2016. ACCEPTED July 11, 2016.

INCLUDE WHEN CITING Published online September 30, 2016; DOI: 10.3171/2016.7.PEDS16310. 
velopment following SRC. $8,18,21,26,27,29,35$ While early identification of these risk factors may be important to help provide anticipatory guidance for patients and parents regarding expected length of recovery, ${ }^{26,27,41}$ understanding the relationship between these clinical variables and the pathophysiology of SRC may provide insight into how these variables contribute to the development of PCS.

In a previous study, we demonstrated that $29 \%$ of pediatric patients with acute SRC and 63\% of those with PCS referred to a multidisciplinary pediatric concussion program demonstrated subjective and objective evidence of vestibulo-ocular dysfunction (VOD) at initial consultation..$^{12}$ Patients with VOD took twice as long to achieve clinical recovery compared with those without VOD and were 4 times more likely to develop PCS, even after we controlled for the confounding effects of other variables identified in the literature. Although these findings provide insight into one of the pathophysiological mechanisms mediating PCS and help identify those patients who may benefit from targeted rehabilitative strategies, this study did not examine the clinical predictors of VOD among this unique population.

Accordingly, the primary objective of this study was to examine the clinical variables associated with the presence of VOD at initial presentation among patients referred to a multidisciplinary pediatric concussion program. The secondary objectives were to reevaluate the prevalence of VOD among this clinical cohort and examine the effect of VOD on length of recovery and the development of PCS.

\section{Methods}

\section{Research Design and Participants}

We performed a retrospective chart review of all consecutive pediatric patients with an SRC referred to the Pan Am Concussion Program at Pan Am Clinic, Winnipeg, Manitoba, Canada, from September 1, 2013, to May 25, 2015. The Pan Am Concussion Program is a multidisciplinary concussion program that accepts referrals for children (19 years old or younger) with acute sports- and non-sports-related concussions and PCS from Manitoba, Northwestern Ontario, and Saskatchewan. Study inclusion criteria included 1) age 19 or younger, and 2) diagnosis of acute SRC or 3) diagnosis of PCS following SRC. All sports were included; exclusion criteria were 1) the presence of traumatic abnormalities on neuroimaging or traumatic structural cervical spine injury; 2) diagnosis of a second concussion during follow-up for a previous symptomatic concussion; or 3) diagnosis of coexistent or previously diagnosed neuroophthalmological conditions (such as strabismus or cranial neuropathy). If an athlete suffered a concussion, recovered, and returned to play and then suffered a second concussion, only the first concussion was included in the analysis. This retrospective study was approved by the institutional ethics review board at the University of Manitoba. Some patients $(n=151)$ were included in a previously reported study. ${ }^{12}$

\section{Definitions}

Sports-related concussion was defined according to the International Consensus on Concussion in Sport as an injury caused by transmission of biomechanical forces to the brain leading to clinical symptoms affecting multiple domains of physical, cognitive, sleep, and neurobehavioral functioning. ${ }^{24}$ To compare findings to those of our previous study, ${ }^{12}$ we defined acute SRC as a clinical consultation on a patient evaluated less than 30 days from the time of injury. Previous authors have pointed out the lack of consensus regarding a universal definition of PCS. ${ }^{41}$ In this study, patients were diagnosed with PCS by the neurosurgeon if they endorsed 3 or more postconcussion symptoms identified by the International Classification of Diseases, 10th Revision (ICD-10) definition ${ }^{6}$ and remained symptomatic at 1 month (30 days) postinjury or longer.

At the time of this study, the authors were aware of only 1 standardized definition of VOD. In accordance with our previous study, ${ }_{13}$ we defined VOD as those patients with more than 1 subjective complaint of intermittent blurred or double vision; visual disturbance; difficulty concentrating, focusing, or reading; dizziness; or motion sensitivity, and the presence of more than 1 of the following objective physical examination findings: abnormal near-point convergence (NPC), abnormal extraocular movements or smooth pursuits, or abnormal or symptomatic assessment of horizontal saccades, vertical saccades, or vestibulo-ocular reflexes (VORs). ${ }^{12}$ The presence of VOD was assessed at initial consultation for all patients with acute SRC and PCS. In general, patients were classified as fully recovered when they were asymptomatic at rest according to clinical interview and the Post-Concussion Symptom Scale (PCSS), were asymptomatic during full-time school activities, completed the International Consensus on Concussion in Sport graduated return-to-play protocol, ${ }^{24}$ and did not meet the clinical criteria for VOD. In cases in which patients harbored preexisting conditions such as migraine headaches, depression, and other preinjury conditions associated with concussion symptoms, patients were classified as fully recovered when they felt they had returned to their neurological baseline, were tolerating full-time school without symptom exacerbation, and had completed the graduated return-to-play guidelines without symptom exacerbation. In some cases, graded aerobic treadmill testing and neuropsychological testing were used to confirm recovery in these patients at the discretion of the treatment team. To assess the effect of clinical variables on the development of PCS, acute SRC patients were dichotomized into 2 groups: 1) those patients who developed PCS during follow-up, and 2) those who did not develop PCS during follow-up.

\section{Clinical Assessments and Management}

At the time of initial medical consultation all patients completed a standardized data collection form that included demographic data, past medical and concussion history, family history, and information regarding the symptoms experienced at the time of injury and whether the patient sustained a loss of consciousness (LOC) or experienced posttraumatic amnesia at the time of injury. At initial consultation, all patients completed the PCSS, a self-reported symptom inventory that includes 22 symp- 
toms rated on a 7-point (0-6) Likert scale with a maximum score of 132 .

All patients underwent a clinical history and physical examination by a single neurosurgeon. The physical examination included a standardized, focused vestibulo-ocular examination that evaluated gross extraocular movements and smooth pursuits, NPC, horizontal and vertical saccades, and VOR using previously described techniques. ${ }^{12}$

Specifically, ocular smooth pursuits were assessed by having the patient follow a slowly moving object through all quadrants of his or her visual field. An abnormal test was indicated by the inability to follow the object without saccadic eye movements. NPC was tested by having the patient fixate on a target that was moved approximately 1-2 cm per second toward the patient's eyes in the midline. An abnormal test was defined as the development of diplopia or inability of the eyes to maintain fusion at a distance greater than $6 \mathrm{~cm}$ from the bridge of the patient's nose. Horizontal and vertical saccades were tested by having the patient look quickly back and forth between 2 targets positioned $30^{\circ}$ from midline in the horizontal and vertical planes, respectively. An abnormal test was defined by the presence of overshooting or more than 2 saccadic corrections during testing. Testing of horizontal saccades and vertical saccades were classified as normal or abnormal, based on examination findings and symptomatic (defined as eliciting or worsening vestibular and oculomotor symptoms) or asymptomatic (defined as eliciting no symptoms). VOR testing was also completed using a modified head-shaking test, whereby the patient was asked to fixate on an object $0.5-1$ meter from the bridge of the nose and rapidly, but comfortably, shake their head back and forth $30^{\circ}$ from midline for $5-10$ seconds. An abnormal test was defined as eliciting or worsening of vestibular and oculomotor symptoms. The head thrust test was also used to evaluate VOR functioning in select patients. However, because it was not performed in all patients in this cohort, test results were not incorporated into the clinical definition of VOD. The focused vestibulo-ocular examination techniques used here are commonly used among neuroophthalmologists, ${ }^{37}$ and an international working group of concussion experts and vestibular therapists rated most of these techniques as demonstrating strong clinical utility for confirming CNS vestibular and oculomotor dysfunction in patients with concussion. ${ }^{32}$ Referrals for vestibular physiotherapy were conducted for patients as indicated by the neurosurgeon. In general, patients with acute SRC who demonstrated clinical evidence of VOD that persisted at 2-4 weeks postinjury, and patients with PCS who demonstrated VOD at initial assessment, were considered for referral to the vestibular physiotherapist.

\section{Statistical Analysis}

Baseline characteristics for all patients were summarized using proportions for dichotomous/polytomous characteristics and means with standard deviations for continuous characteristics. If a continuous variable was not normally distributed, it was summarized as a median with an interquartile range (IQR). The Student t-test was used to compare means between groups, and Pearson's chi-square and Fisher's exact tests were used for comparisons of categorical variables where appropriate. Backward elimination Poisson regression analysis was used to identify factors that increased or decreased the risk (with 95\% confidence intervals [CIs]) of developing VOD. ${ }^{25}$ Variables were included if they were statistically significant in the univariate analysis. In the multivariate model, variables that were not significant risk factors were assessed as confounders. If none of the risk ratios (RRs) of the significant risk factors changed by less than $15 \%$, the variable was not considered to confound the association between risk factors and outcome. ${ }^{28}$

Poisson regression was also used to identify significant predictors of PCS among those who presented with an acute SRC. Clinical predictors of PCS that were previously identified in the literature and included in the analysis were: age, sex, preinjury history of attention deficit hyperactivity disorder (ADHD), history of learning disorder, history of depression, history of headaches or migraine headaches, history of previous concussion, LOC, posttraumatic amnesia, and VOD. Although initial PCSS score has been identified as a predictor of prolonged recovery in previous studies, we chose not to include this variable in the multivariate model for two important reasons: 1) baseline PCSS scores were not available for patients included in this study so it was impossible to evaluate the independent effect of injury on initial PCSS score, apart from other baseline conditions that can elevate these scores in adolescents (preinjury depression, migraine, etc.): ${ }^{17}$ and 2 ) we hypothesized that patients with acute SRC with VOD and those with a preinjury history of migraine and depression would report higher initial PCSS scores, and that a strong association between the presence of these factors and initial PCSS would not permit accurate assessment of the independent effect of these clinical variables on length of recovery and the development of PCS. Statistical significance was set at $\mathrm{p}<0.05$. Therapeutic interventions such as vestibular physiotherapy were tabulated. All statistical analyses were performed using Stata (version 12.1, Stata Corp.).

\section{Results}

\section{Participants and Prevalence of VOD}

During the study period, 399 patients met the inclusion criteria for the study including $306(76.7 \%)$ with acute SRC and 93 (23.3\%) with PCS (Table 1). Of the 306 patients with acute SRC $(65.0 \%$ male, mean age $13.9 \pm$ 2.3 years old), 92 (30.1\%) met the criteria for VOD. For patients with acute SRC, the median time from injury to initial consultation was 7 days (IQR 5-12 days). Of the 93 patients who presented with PCS (41.9\% male, mean age $15.4 \pm 2.0$ years), $40(43.0 \%)$ met the criteria for VOD. For patients with PCS the median time from injury to initial consultation was 86 days (IQR 41-150 days). For all patients included in the study, hockey $(\mathrm{n}=171)$ and soccer (n $=58$ ) were the most commonly played sports at the time of injury. Additional characteristics of the study cohort are shown in Table 1.

\section{Clinical Predictors of VOD}

Univariate analysis revealed several clinical variables that were associated with VOD among all study patients 
TABLE 1. Baseline characteristics of patients with acute SRC and PCS

\begin{tabular}{|c|c|c|c|c|}
\hline Variable & All Concussions (\%) & Acute SRC (\%) & PCS (\%) & $\mathrm{p}$ Value \\
\hline No. of patients & 399 & $306(76.7)$ & $93(23.3)$ & \\
\hline Mean age in yrs \pm SD & $14.3 \pm 2.3$ & $13.9 \pm 2.3$ & $15.4 \pm 2.0$ & \\
\hline Males & $238(59.7)$ & $199(65.0)$ & $39(41.9)$ & $<0.0001$ \\
\hline History of prior concussion & $185(46.4)$ & $129(42.2)$ & $56(60.2)$ & 0.002 \\
\hline History of headaches or migraine headaches & $43(10.8)$ & $25(8.2)$ & $18(19.4)$ & 0.002 \\
\hline LOC & $64(16.0)$ & $42(13.7)$ & $22(23.7)$ & 0.024 \\
\hline Missing & $7(1.8)$ & $6(2.0)$ & $1(1.1)$ & \\
\hline Posttraumatic amnesia & $127(31.8)$ & $94(30.7)$ & $33(35.5)$ & 0.388 \\
\hline Delayed symptoms & $119(29.8)$ & $85(27.8)$ & $34(36.6)$ & 0.105 \\
\hline Median initial PCSS score at consultation, IQR & $13,3.0-31.0$ & $10,1.8-29.0$ & $23,9.3-39.5$ & $<0.0001$ \\
\hline VOD at consultation & $132(33.1)$ & $92(30.1)$ & $40(43.0)$ & 0.02 \\
\hline
\end{tabular}

Boldface type indicates statistical significance.

(Table 2). On multivariate analysis, significant predictors of VOD included dizziness at injury (RR $1.95,95 \%$ CI $1.06-3.59$ ), preinjury history of depression (RR $1.53,95 \%$ CI 1.03-2.29), female sex (RR 1.45, 95\% CI 1.05-2.01), difficulty focusing at injury (RR $1.43,95 \%$ CI 1.00-2.05), blurred vision at injury (RR 1.42, 95\% CI 1.01-1.99), and posttraumatic amnesia (RR 1.39, 95\% CI 1.02-1.91). Patients who presented with acute SRC and VOD had a significantly higher PCSS score (median 32, IQR 17-50) than patients with acute SRC without VOD (median 5, IQR $0-15, \mathrm{p}<0.0001)$. Patients with acute SRC and a preinjury history of depression demonstrated higher initial PCSS scores (median 39, IQR 22-50) than patients with acute

TABLE 2. Univariate analysis of clinical variables associated with the development of VOD among those with acute SRC

\begin{tabular}{lcc}
\hline \multicolumn{1}{c}{ Variable } & $\mathrm{RR}(95 \% \mathrm{Cl})$ & $\mathrm{p} \mathrm{Value}$ \\
\hline Age & $1.10(1.02-1.18)$ & $\mathbf{0 . 0 1 8}$ \\
\hline Males & $0.61(0.44-0.86)$ & $\mathbf{0 . 0 0 4}$ \\
\hline History of prior concussion & $1.20(0.86-1.70)$ & 0.286 \\
\hline $\begin{array}{l}\text { History of headaches or } \\
\text { migraine headaches }\end{array}$ & $0.65(0.29-1.44)$ & 0.287 \\
\hline History of motion sickness & $0.97(0.51-1.85)$ & 0.921 \\
\hline LOC & $1.13(0.71-1.81)$ & 0.605 \\
\hline Posttraumatic amnesia & $1.52(1.08-2.13)$ & $\mathbf{0 . 0 1 6}$ \\
\hline Delayed symptoms & $1.32(0.93-1.88)$ & 0.122 \\
\hline History of ADHD & $1.51(0.91-2.48)$ & 0.113 \\
\hline History of learning disorder & $1.26(0.50-3.13)$ & 0.625 \\
\hline History of depression & $2.45(1.49-4.05)$ & $<0.001$ \\
\hline Symptoms at injury & & \\
\hline Headache & $1.75(0.61-4.95)$ & 0.295 \\
\hline Dizziness & $2.62(1.43-4.78)$ & $\mathbf{0 . 0 0 2}$ \\
\hline Imbalance & $1.60(1.14-2.25)$ & $\mathbf{0 . 0 0 7}$ \\
\hline Difficulty focusing & $2.00(1.39-2.89)$ & $<0.001$ \\
\hline Blurred vision & $1.89(1.35-2.65)$ & $<0.001$ \\
\hline Nausea & $1.46(1.03-2.07)$ & $\mathbf{0 . 0 3 1}$ \\
\hline Vomiting & $0.86(0.42-1.75)$ & 0.674 \\
\hline Bordace & &
\end{tabular}

Boldface type indicates statistical significance.
SRC and no previous depression (median 10, IQR 1-28; $\mathrm{p}=0.032$ ).

\section{Clinical Predictors of PCS}

Among the patients with acute SRC, 122 (40.1\%) developed PCS. The median length of recovery among patients with acute SRC and VOD was 40 days (IQR 24-70 days) compared with 20 days (IQR 12-28 days) among those without VOD ( $\mathrm{p}<0.0001)$. Table 3 describes the baseline characteristics of those who presented with an acute SRC and subsequently developed or did not develop PCS. Univariate analysis revealed several variables that were significantly associated with the development of PCS among patients with acute SRC (Table 4). On multivariate analysis, VOD (RR 2.61, 95\% CI 2.00-3.41), posttraumatic amnesia (RR 1.41, 95\% CI 1.10-1.80), and preinjury history of depression (RR 1.50, 95\% CI 1.09-2.05) were found to be independent predictors of PCS. Age, sex, previous diagnosis of ADHD, previous concussions, LOC, or previous diagnosis of learning disorders did not confound any of the observed associations. Sex was not significantly associated with past depression (RR 4.64, 95\% CI 0.75-28.59) among those with acute SRC. Although there was more power to detect a significant association with sex and PCS than past depression and PCS, sex was never a significant predictor of PCS when sex replaced past depression in the modeling process. Sex did not confound the final model that included past depression, as the estimate changed by less than $10 \%(8.5 \%)$.

\section{Interventions and Outcomes}

Overall, 132 patients $(33.1 \%)$ met the clinical criteria for VOD in this study and 56 underwent vestibular physiotherapy (22 patients with acute SRC and 34 with PSC). Of the 22 patients with acute SRC who were referred for vestibular physiotherapy, 11 initiated treatment after meeting the criteria for PCS and 11 initiated before meeting the criteria for PCS. Among the 306 patients with acute SRC, 31 were lost to follow-up prior to medical clearance and 251 received medical clearance within the study period (median 22 days, IQR 15-35 days) while 24 patients remained under the medical care of the treat- 
TABLE 3. Comparison of patients with acute SRC who did and did not develop PCS

\begin{tabular}{lcccc}
\hline & All Acute & \multicolumn{2}{c}{ Developed PCS } & P Value \\
\cline { 5 - 5 } \multicolumn{1}{c}{ Variable } & SRC $(\%)^{*}$ & No $(\%)$ & Yes $(\%)$ & p \\
\hline No. of patients & 306 & 182 & 122 & \\
\hline Mean age in yrs \pm SD & $13.92 \pm 2.3$ & $13.7 \pm 2.5$ & $14.3 \pm 2.0$ & 0.9855 \\
\hline Males & $199(65.0)$ & $129(70.9)$ & $69(56.6)$ & 0.010 \\
\hline History of prior concussion & $129(42.2)$ & $75(41.2)$ & $53(43.4)$ & 0.699 \\
\hline History of headaches or migraine headaches & $25(8.2)$ & $14(7.7)$ & $11(9.0)$ & 0.680 \\
\hline LOC & $42(13.7)$ & $27(14.8)$ & $15(12.3)$ & 0.611 \\
\hline Missing & $6(2.0)$ & $1(0.6)$ & $5(4.1)$ & \\
\hline Posttraumatic amnesia & $94(30.7)$ & $42(23.1)$ & $51(41.8)$ & 0.001 \\
\hline Delayed symptoms & $85(27.8)$ & $46(25.3)$ & $39(32.0)$ & 0.203 \\
\hline Median initial PCSS score at consultation, IQR & $10,1.8-29.0$ & $4,0-13$ & $26,14-44$ & $<0.0001$ \\
\hline VOD at consultation & $92(30.1)$ & $25(13.7)$ & $67(54.9)$ & $<0.0001$ \\
\hline
\end{tabular}

Boldface type indicates statistical significance.

* There was incomplete follow-up data available for 2 patients with acute SRC.

ing medical team. Of the 93 patients with PCS, 38 were lost to follow-up prior to medical clearance, 30 received medical clearance within the study period (median 123 days, IQR 88-238 days), and 25 remained under the care of the treating medical team.

\section{Discussion}

To our knowledge, this is the first study to identify clinical risk factors of VOD at initial consultation among pediatric patients with SRC. Patients with acute SRC who were female, had a preinjury history of depression, or who experienced posttraumatic amnesia, dizziness, blurred vision, and difficulty focusing at the time of injury were significantly more likely to demonstrate clinical evidence of VOD at initial consultation. Previous work has demonstrated that athletes who experience dizziness at the time of injury are at an elevated risk of protracted recovery following SRC. ${ }^{19}$ The association between female sex and preinjury history of depression with the finding of VOD at initial consultation in this study is difficult to account for. Previous studies have suggested that females are at a higher risk of concussion and are at an elevated risk of prolonged recovery following concussion as compared with their male counterparts. ${ }^{9,10}$ One of the mechanisms thought to account for sex differences in concussion is the strength of the cervical spine musculature and its effect on buffering biomechanical forces that are applied to the head and neck at the time of injury. ${ }^{36}$ Although neck strength was not formally assessed in this study, it is possible that weaker cervical spine musculature among females leads to the transmission of greater biomechanical forces to the brain and neural substrates of vestibular and oculomotor function, thereby increasing the risk of VOD. In general, females have been found to report more symptoms after concussion than males, ${ }^{3,7}$ which may have made them more likely to report subjective symptoms of VOD or endorse symptom provocation during objective vestibulo-ocular examination in this study. The relationship between VOD and injury severity is supported by the finding that posttraumatic amnesia, a marker of injury severity, was also associated with the presence of VOD. As well, patients with VOD endorsed a higher number of symptoms than those without VOD, which may also have indicated more severe injuries. Indeed, patients with acute SRC and VOD were found to have a median initial PCSS score of $32 \mathrm{com}$ pared with a score of 5 for patients without VOD. Previous studies have suggested that depression and other mood disorders are also associated with objective abnormalities in saccades, smooth pursuits, and visual processing. ${ }^{5,33,38}$ Therefore, future studies are needed to evaluate whether

TABLE 4. Univariate analysis of clinical variables associated with the development of PCS

\begin{tabular}{|c|c|c|}
\hline Variable & $\operatorname{RR}(95 \% \mathrm{Cl})$ & $p$ Value \\
\hline Age & $1.07(1.01-1.14)$ & 0.024 \\
\hline Males & $0.70(0.53-0.91)$ & 0.009 \\
\hline History of prior concussion & $1.06(0.80-1.39)$ & 0.699 \\
\hline $\begin{array}{l}\text { History of headaches or } \\
\text { migraine headaches }\end{array}$ & $1.11(0.69-1.76)$ & 0.672 \\
\hline History of motion sickness & $1.16(0.73-1.83)$ & 0.536 \\
\hline LOC & $0.90(0.58-1.38)$ & 0.621 \\
\hline Posttraumatic amnesia & $1.63(1.25-2.12)$ & $<0.0001$ \\
\hline Delayed symptoms & $1.21(0.91-1.61)$ & 0.192 \\
\hline Past ADHD & $1.40(0.93-2.11)$ & 0.108 \\
\hline History of learning disorder & $1.58(0.91-2.76)$ & 0.106 \\
\hline History of depression & $2.19(1.57-3.07)$ & $<0.0001$ \\
\hline \multicolumn{3}{|l|}{ Symptoms at injury } \\
\hline Headache & $1.75(0.73-4.17)$ & 0.208 \\
\hline Dizziness & $1.64(1.10-2.45)$ & 0.016 \\
\hline Imbalance & $1.04(0.78-1.37)$ & 0.804 \\
\hline Difficulty focusing & $1.49(1.12-1.97)$ & 0.006 \\
\hline Blurred vision & $1.41(1.07-1.84)$ & 0.013 \\
\hline Nausea & $1.18(0.90-1.55)$ & 0.240 \\
\hline Vomiting & $0.74(0.39-1.40)$ & 0.360 \\
\hline VOD & $2.81(2.16-3.64)$ & $<0.0001$ \\
\hline
\end{tabular}

Boldface type indicates statistical significance. 
baseline characteristics of vestibulo-ocular function among females and those with depression place these populations at an elevated risk of developing VOD following SRC.

The results of this study are consistent with our previous study ${ }^{12}$ and provide confirmatory evidence that VOD is commonly detected among pediatric patients with acute SRC and PCS and is associated with prolonged recovery and an elevated risk of developing PCS. Overall, VOD was detected at initial consultation in $30 \%$ of patients with acute SRC and $43 \%$ of patients with PCS. The median length of recovery among patients with acute SRC and VOD was 40 days compared with 20 days among patients without VOD. On multivariate analysis, the presence of VOD at initial consultation, a preinjury history of depression, and posttraumatic amnesia were found to be independent predictors of developing PCS following acute SRC. These results are in agreement with other studies that have demonstrated alterations in accommodation, convergence, and saccades following concussion ${ }^{22,30,31}$ and 1 study that found that abnormal convergence was associated with prolonged recovery following pediatric concussion. ${ }^{8}$ Previous studies have also suggested that a preinjury mood disorder $^{8,29}$ and posttraumatic amnesia ${ }^{23}$ are independent predictors of longer recovery following SRC.

Although the focused vestibulo-ocular physical examination used in this study may help identify concussion patients who are at risk for PCS, the use of these tools in isolation by healthcare professionals without training in traumatic brain injury may permit other serious causes of visual disturbance to go undiagnosed among these patients. This is exemplified by the accumulating number of reported cases of patients who presented with visual and oculomotor disturbance following minor head injury and SRC that were found to be caused by coexisting traumatic cranial neuropathy, ${ }^{14,15}$ demyelinating disease, ${ }^{13}$ paraneoplastic syndrome, ${ }^{39}$ and retinal detachment. ${ }^{4}$ Accordingly, the authors suggest that these vestibulo-ocular tests only be used to supplement a full neurological examination and only be performed by clinicians who are trained to recognize the full spectrum of neuroophthalmological conditions that can accompany traumatic brain injury.

In recent years, vestibular physiotherapy has emerged as a promising therapeutic intervention to help target the pathophysiological mechanisms governing VOD after concussion. ${ }^{1,34}$ In this study, $42 \%$ of patients with SRC and VOD detected at initial consultation were treated with targeted vestibular rehabilitation. Based on the retrospective nature of this study and the absence of a control group, no conclusions can be made regarding the therapeutic benefit of vestibular therapy in this clinical population. Consequently, future prospective studies remain needed to determine appropriate clinical indications for targeted vestibular physiotherapy and assess the effect of treatment in these patients.

The results of this study must be interpreted in light of several limitations. First, this study included children and adolescents referred to a tertiary multidisciplinary concussion program that may have selected for patients with more severe injuries and those who were more likely to develop PCS. Second, although patients with acute SRC were evaluated 7 days postinjury (on average), this study did include patients with varying levels of acuity. Therefore, the study findings are applicable to patients who are often evaluated days after injury at tertiary concussion clinics but may not be generalizable to more acute patients evaluated on the sidelines or in the emergency department setting. Third, although the definitions used to classify different components of the focused vestibulo-ocular examination in this study have been described previously in the literature, it is likely that developmental changes in ocular alignment, and oculomotor and vestibular functioning, continue throughout childhood and adolescence. As such, some of the observed findings on physical examination may have been classified as abnormal by these definitions but may have indeed been normal for that individual and not independently responsible for the patient's subjective vestibulo-ocular symptoms. Some of the vestibulo-ocular symptoms (such as dizziness) may have been attributable to the effects of a global metabolic brain injury or coexisting cervical spine soft-tissue injury and not isolated dysfunction within the vestibulo-ocular system. These subtleties underscore the need for incorporation of validated objective oculomotor and vestibular outcome measures in future studies and have important implications for confirming the diagnosis of selected neuroophthalmological conditions (i.e., convergence insufficiency) as well as developing evidence-based indications for targeted rehabilitative strategies. Fourth, a significant proportion of patients with VOD in this study did undergo vestibular physiotherapy, which may have ameliorated some of the effects of VOD on PCS development and recovery time among those patients. Lastly, the neurosurgeon was not blinded to the patient's presenting symptoms, but the same clinical assessment and physical examination was performed on all patients regardless of symptoms.

\section{Conclusions}

This study is the first to demonstrate that female sex, preinjury history of depression, or posttraumatic amnesia, dizziness, blurred vision, and difficulty focusing at the time of injury are independent risk factors for the development of VOD among pediatric patients with SRC. Patients with VOD take twice as long to recover following acute SRC compared with those without VOD. Finally, the presence of VOD at initial consultation, a preinjury history of depression, and posttraumatic amnesia at the time of injury are independent risk factors for the development of PCS following pediatric SRC. Future studies examining clinical decision-making rules to predict recovery following concussion should include the role of VOD. A prospective study to evaluate the natural history of VOD following pediatric SRC and establish evidence-based clinical indications for targeted vestibular physiotherapy will be undertaken at the authors' institution.

\section{Acknowledgments}

All phases of this study were supported by a grant from the Pan Am Clinic Foundation, Winnipeg Jets True North Foundation, and the Manitoba Health Research Council.

\section{References}

1. Alsalaheen BA, Mucha A, Morris LO, Whitney SL, Furman 
JM, Camiolo-Reddy CE, et al: Vestibular rehabilitation for dizziness and balance disorders after concussion. J Neurol Phys Ther 34:87-93, 2010

2. Babcock L, Byczkowski T, Wade SL, Ho M, Mookerjee S, Bazarian JJ: Predicting postconcussion syndrome after mild traumatic brain injury in children and adolescents who present to the emergency department. JAMA Pediatr 167:156161,2013

3. Baker JG, Leddy JJ, Darling SR, Shucard J, Makdissi M, Willer BS: Gender differences in recovery from sportsrelated concussion in adolescents. Clin Pediatr (Phila) 55:771-775, 2016

4. Bedgood A, Rand SE, Major J Jr: Occult retinal detachment after mild traumatic brain injury: case report and literature review. Clin J Sport Med 25:e26-e27, 2015

5. Bittencourt J, Velasques B, Teixeira S, Basile LF, Salles JI, Nardi AE, et al: Saccadic eye movement applications for psychiatric disorders. Neuropsychiatr Dis Treat 9:1393-1409, 2013

6. Boake C, McCauley SR, Levin HS, Contant CF, Song JX, Brown SA, et al: Limited agreement between criteria-based diagnoses of postconcussional syndrome. J Neuropsychiatry Clin Neurosci 16:493-499, 2004

7. Colvin AC, Mullen J, Lovell MR, West RV, Collins MW, Groh M: The role of concussion history and gender in recovery from soccer-related concussion. Am J Sports Med 37:1699-1704, 2009

8. Corwin DJ, Zonfrillo MR, Master CL, Arbogast KB, Grady MF, Robinson RL, et al: Characteristics of prolonged concussion recovery in a pediatric subspecialty referral population. J Pediatr 165:1207-1215, 2014

9. Covassin T, Swanik CB, Sachs ML: Sex differences and the incidence of concussions among collegiate athletes. J Athl Train 38:238-244, 2003

10. Dick RW: Is there a gender difference in concussion incidence and outcomes? Br J Sports Med 43 (Suppl 1):i46i50, 2009

11. Eisenberg MA, Meehan WP III, Mannix R: Duration and course of post-concussive symptoms. Pediatrics 133:9991006, 2014

12. Ellis MJ, Cordingley D, Vis S, Reimer K, Leiter J, Russell K: Vestibulo-ocular dysfunction in pediatric sports-related concussion. J Neurosurg Pediatr 16:248-255, 2015

13. Ellis MJ, Leddy JJ, Willer B: Physiological, vestibulo-ocular and cervicogenic post-concussion disorders: an evidencebased classification system with directions for treatment. Brain Inj 29:238-248, 2015

14. Ellis MJ, McDonald PJ, Cordingley D, Mansouri B, Essig M, Ritchie L: Retirement-from-sport considerations following pediatric sports-related concussion: case illustrations and institutional approach. Neurosurg Focus 40(4):E8, 2016

15. Ellis MJ, Ritchie L, Cordingley D, Essig M, Mansouri B: Traumatic optic neuropathy: a potentially unrecognized diagnosis after sports-related concussion. Curr Sports Med Rep 15:27-32, 2016

16. Grubenhoff JA, Deakyne SJ, Brou L, Bajaj L, Comstock RD, Kirkwood MW: Acute concussion symptom severity and delayed symptom resolution. Pediatrics 134:54-62, 2014

17. Iverson GL, Silverberg ND, Mannix R, Maxwell BA, Atkins JE, Zafonte R, et al: Factors associated with concussion-like symptom reporting in high school athletes. JAMA Pediatr 169:1132-1140, 2015

18. Kutcher JS, Eckner JT: At-risk populations in sports-related concussion. Curr Sports Med Rep 9:16-20, 2010

19. Lau BC, Kontos AP, Collins MW, Mucha A, Lovell MR: Which on-field signs/symptoms predict protracted recovery from sport-related concussion among high school football players? Am J Sports Med 39:2311-2318, 2011

20. Makdissi M, Cantu RC, Johnston KM, McCrory P, Meeuwisse WH: The difficult concussion patient: what is the best approach to investigation and management of persistent $(>10$ days) postconcussive symptoms? Br J Sports Med 47:308313, 2013

21. Makdissi M, Davis G, Jordan B, Patricios J, Purcell L, Putukian M: Revisiting the modifiers: how should the evaluation and management of acute concussions differ in specific groups? Br J Sports Med 47:314-320, 2013

22. Master CL, Scheiman M, Gallaway M, Goodman A, Robinson RL, Master SR, et al: Vision diagnoses are common after concussion in adolescents. Clin Pediatr (Phila) 55:260-267, 2016

23. McCrea M, Guskiewicz K, Randolph C, Barr WB, Hammeke TA, Marshall SW, et al: Incidence, clinical course, and predictors of prolonged recovery time following sport-related concussion in high school and college athletes. J Int Neuropsychol Soc 19:22-33, 2013

24. McCrory P, Meeuwisse WH, Aubry M, Cantu B, Dvorák J, Echemendia RJ, et al: Consensus statement on concussion in sport: the 4th International Conference on Concussion in Sport held in Zurich, November 2012. Br J Sports Med 47:250-258, 2013

25. McNutt LA, Wu C, Xue X, Hafner JP: Estimating the relative risk in cohort studies and clinical trials of common outcomes. Am J Epidemiol 157:940-943, 2003

26. Meehan WP III, Mannix R, Monuteaux MC, Stein CJ, Bachur RG: Early symptom burden predicts recovery after sport-related concussion. Neurology 83:2204-2210, 2014

27. Meehan WP III, Mannix RC, Stracciolini A, Elbin RJ, Collins MW: Symptom severity predicts prolonged recovery after sport-related concussion, but age and amnesia do not. J Pediatr 163:721-725, 2013

28. Mickey RM, Greenland S: The impact of confounder selection criteria on effect estimation. Am J Epidemiol 129:125137, 1989

29. Morgan CD, Zuckerman SL, Lee YM, King L, Beaird S, Sills AK, et al: Predictors of postconcussion syndrome after sports-related concussion in young athletes: a matched casecontrol study. J Neurosurg Pediatr 15:589-598, 2015

30. Mucha A, Collins MW, Elbin RJ, Furman JM, TroutmanEnseki C, DeWolf RM, et al: A Brief Vestibular/Ocular Motor Screening (VOMS) assessment to evaluate concussions: preliminary findings. Am J Sports Med 42:2479-2486, 2014

31. Pearce KL, Sufrinko A, Lau BC, Henry L, Collins MW, Kontos AP: Near point of convergence after a sport-related concussion: measurement reliability and relationship to neurocognitive impairment and symptoms. Am J Sports Med 43:3055-3061, 2015

32. Reneker JC, Clay Moughiman M, Cook CE: The diagnostic utility of clinical tests for differentiating between cervicogenic and other causes of dizziness after a sports-related concussion: An international Delphi study. J Sci Med Sport 18:366-372, 2015

33. Rommelse NN, Van der Stigchel S, Sergeant JA: A review on eye movement studies in childhood and adolescent psychiatry. Brain Cogn 68:391-414, 2008

34. Schneider KJ, Meeuwisse WH, Nettel-Aguirre A, Barlow K, Boyd L, Kang J, et al: Cervicovestibular rehabilitation in sport-related concussion: a randomised controlled trial. Br J Sports Med 48:1294-1298, 2014

35. Scopaz KA, Hatzenbuehler JR: Risk modifiers for concussion and prolonged recovery. Sports Health 5:537-541, 2013

36. Tierney RT, Sitler MR, Swanik CB, Swanik KA, Higgins M, Torg J: Gender differences in head-neck segment dynamic stabilization during head acceleration. Med Sci Sports Exerc 37:272-279, 2005

37. Ventura RE, Balcer LJ, Galetta SL: The neuro-ophthalmology of head trauma. Lancet Neurol 13:1006-1016, 2014

38. Winograd-Gurvich C, Georgiou-Karistianis N, Fitzgerald PB, Millist L, White OB: Self-paced and reprogrammed sac- 
cades: differences between melancholic and non-melancholic depression. Neurosci Res 56:253-260, 2006

39. Wu H, Mody AP: A toddler with uncontrollable shaking after a minor fall. Pediatr Emerg Care [epub ahead of print], 2015

40. Yeates KO, Luria J, Bartkowski H, Rusin J, Martin L, Bigler ED: Postconcussive symptoms in children with mild closed head injuries. J Head Trauma Rehabil 14:337-350, 1999

41. Zemek RL, Farion KJ, Sampson M, McGahern C: Prognosticators of persistent symptoms following pediatric concussion: a systematic review. JAMA Pediatr 167:259-265, 2013

\section{Disclosures}

The authors report no conflict of interest concerning the materi- als or methods used in this study or the findings specified in this paper.

\section{Author Contributions}

Conception and design: Ellis, Russell. Acquisition of data: all authors. Analysis and interpretation of data: all authors. Drafting the article: Ellis, Russell. Critically revising the article: all authors. Reviewed submitted version of manuscript: all authors. Approved the final version of the manuscript on behalf of all authors: Ellis. Statistical analysis: Russell.

\section{Correspondence}

Michael J. Ellis, Pan Am Clinic, Pan Am Concussion Program, 75 Poseidon Bay, Winnipeg, MB R3M 3E4, Canada. email: mellis3@panamclinic.com. 